\begin{tabular}{|c|c|}
\hline \multirow{3}{*}{ 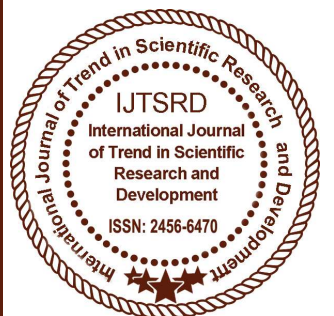 } & $\begin{array}{l}\text { International Journal of Trend in Scientific } \\
\text { Research and Development (IJTSRD) }\end{array}$ \\
\hline & International Open Access Journal \\
\hline & ISSN No: 2456 - 6470 | www.ijtsrd.com | Volume - 2 | Issue - 2 \\
\hline
\end{tabular}

\title{
Concept of Regression Analysis in Concrete Mix Design
}

\author{
Trupti S. Joshi, Manisha S. Kukade, Sumit D. Sanghani \\ U.G. Student, Department of Civil Engineering, Sinhgad Academy of Engineering, \\ Pune, Mahashtra, India
}

\begin{abstract}
In civil engineering, on site concrete mixing is very important .To determine compressive strength of concrete mix, statistical method is very useful. Regression analysis is an effective statistical technique. By analysing the laboratory concrete block/ cylinder tests, we can determine not only the correlation between compressive strength at different curing ages of concrete but also variation in material properties, ratios and variation in $\%$ of fly ash as a partial replacement of concrete.
\end{abstract}

Keywords: Statistical analysis, compression strength ,concrete mix design, fly ash, correlation between compressive strength and days of curing.

\section{INTRODUCTION}

We use concrete as a construction material worldwide. Because of its advantages like low cost, availability of construction, workability, durability and convenient compressive strength. These advantages depend on the concrete mix, placing and curing. In construction industry, strength is a primary criterion in selecting a concrete for a particular application .Concrete used for construction gains strength over a long period of time after pouring. The characteristics strength of concrete is defined as the compressive strength of a sample that has been aged for 90 days.
The aim of the present study is to predict compressive strength of concrete for a given samples made by using industrial waste fly ash as a partial replacement of cement of different hoppers for different concrete grades like M20, M25 and M30 at curing ages of 7, 28 and 90 . And finding correlation between them by using regression analysis.

In this project, we add fly ash as a partial replacement of concrete. And percentage of fly ash varies from $0 \%$ to $37.5 \%$. The work presented in this project reports an investigation on the behaviour of concrete produced from blending cement with FA. Percentages of fly ash are $0 \%, 12.50 \%, 25.00 \%$ and $37.50 \%$ for each hopper of namely 1, 2, 3 for M20, M25 and M30 grades. The utilization of fly ash as cement replacement material in concrete or as additive in cement introduces many benefits from economical, technical and environmental point of view.

\section{EXPERIMENTAL DATA}

Since the aim this study is studying the effect of mix proportions on the compressive strength of concrete, different mixes were used. The details of all mix proportions are shown in Tables below.

Compressive strength test was performed and evaluated. Specimens were immersed in water until the day of testing at 7,28 and 90 days. Tables below show the test results. 
International Journal of Trend in Scientific Research and Development (IJTSRD) ISSN: 2456-6470

TABLE I : PHYSICAL PROPERTIES

\begin{tabular}{|l|c|c|c|c|}
\hline \multicolumn{1}{|c|}{ Test Conducted } & Cement & Hopper No: & Hopper No:2 & Hopper No:3 \\
\hline Specific gravity (gm/cc) & 3.15 & 2.040 & 2.061 & 2.173 \\
\hline $\begin{array}{l}\text { Fineness \% by wt by sieving } \\
\text { (\%retention on 45 micron } \\
\text { sieve-wet sieving) }\end{array}$ & $\ldots$. & 45.55 & 24.45 & 2.70 \\
\hline $\begin{array}{l}\text { Fineness (specific } \\
\text { surface)(Sq.m./kg) By Blains } \\
\text { Air Permeability }\end{array}$ & $\ldots$. & 229 & 320 & 536 \\
\hline
\end{tabular}

QUANTITIES FOR M20 GRADE OF CONCRETE

Same for hopper no 1,2 and 3

\begin{tabular}{|l|c|c|c|c|}
\hline Sr no & $\mathbf{1}$ & $\mathbf{2}$ & $\mathbf{3}$ & $\mathbf{4}$ \\
\hline CEMENT(Kg/m3) & 383 & 383 & 383 & 383 \\
\hline FA(\%) & 00.00 & 12.50 & 25.00 & 37.50 \\
\hline FA(Kg/m3) & 00.00 & 47.87 & 95.75 & 124.47 \\
\hline CEMENT(Kg/m3) & 383.00 & 335.13 & 287.25 & 258.53 \\
\hline SAND(Kg/m3) & 559.00 & 559.00 & 559.00 & 559.00 \\
\hline AGG(Kg/m3) & 1213.00 & 1213.00 & 1213.00 & 1213.00 \\
\hline WATER (Lit) & 186.71 & 186.71 & 186.71 & 186.71 \\
\hline
\end{tabular}

QUANTITIES FOR M25 GRADE OF CONCRETE:

Same for hopper no 1, 2 and 3

\begin{tabular}{|l|l|l|l|l|}
\hline Sr no & 1 & 2 & 3 & 4 \\
\hline CEMENT(Kg/m3) & 456 & 456 & 456 & 456 \\
\hline FA(\%) & 00.00 & 12.50 & 25.00 & 37.50 \\
\hline FA(Kg/m3) & 00.00 & 57.00 & 114.00 & 171.00 \\
\hline CEMENT(Kg/m3) & 456.00 & 399.00 & 342.00 & 285.00 \\
\hline SAND(Kg/m3) & 540.00 & 540.000 & 540.00 & 540.00 \\
\hline AGG(Kg/m3) & 1171.00 & 1171.00 & 1171.00 & 1171.00 \\
\hline WATER (Lit) & 186.877 & 186.877 & 186.877 & 186.877 \\
\hline
\end{tabular}

QUANTITIES FOR M30 GRADE OF CONCRETE

Same for hopper no 1, 2 and 3

\begin{tabular}{|l|l|l|l|l|}
\hline Sr no & $\mathbf{1}$ & $\mathbf{2}$ & $\mathbf{3}$ & $\mathbf{4}$ \\
\hline CEMENT(Kg/m3) & 479 & 479 & 479 & 479 \\
\hline FA(\%) & 00.00 & 12.50 & 25.00 & 37.50 \\
\hline FA(Kg/m3) & 00.00 & 59.875 & 119.75 & 179.625 \\
\hline CEMENT(Kg/m3) & 479.00 & 419.125 & 359.25 & 299.375 \\
\hline SAND(Kg/m3) & 534.00 & 534.00 & 534.00 & 534.00 \\
\hline AGG(Kg/m3) & 1132.00 & 1132.00 & 1132.00 & 1132.00 \\
\hline WATER (Lit) & 186.80 & 186.80 & 186.80 & 186.80 \\
\hline
\end{tabular}


International Journal of Trend in Scientific Research and Development (IJTSRD) ISSN: 2456-6470

\section{III.RESULTS AND DISCUSSION}

FOR HOPPER NO:1 [M20 GRADE]

\begin{tabular}{|l|c|c|c|c|}
\hline Sr. No. & $\mathbf{1}$ & $\mathbf{2}$ & $\mathbf{3}$ & $\mathbf{4}$ \\
\hline 7 Day & 20.15 & 18.22 & 17.92 & 17.92 \\
\hline 28 Day & 27.71 & 23.50 & 24.67 & 22.97 \\
\hline 90Day & 28.30 & 26.23 & 28.89 & 25.34 \\
\hline $\begin{array}{l}\text { Square of Coeff. } \\
\text { of Correlation }\end{array}$ & 0.548 & 0.803 & 0.841 & 0.784 \\
\hline Equation & $\begin{array}{l}\mathrm{Y}=0.07 \\
8 \mathrm{X}+22 .\end{array}$ & $\begin{array}{l}\mathrm{Y}=0.08 \\
4 \mathrm{X}+19 .\end{array}$ & $\begin{array}{l}\mathrm{Y}=0.117 \mathrm{X} \\
+18.92\end{array}$ & $\begin{array}{l}\mathrm{Y}=0.077 \mathrm{X} \\
+18.83\end{array}$ \\
\hline $\begin{array}{l}13 \\
\text { Prediscted C.S. }\end{array}$ & 24.314 & 21.472 & 22.196 & 20.986 \\
\hline
\end{tabular}

FOR HOPPER NO:1 [M25 GRADE]

\begin{tabular}{|l|c|c|c|c|}
\hline Sr. No. & $\mathbf{5}$ & $\mathbf{6}$ & $\mathbf{7}$ & $\mathbf{8}$ \\
\hline 7 Day & 21.63 & 17.63 & 17.92 & 18.82 \\
\hline 28 Day & 35.85 & 30.82 & 31.41 & 26.52 \\
\hline 90Day of Coeff. & 36.89 & 29.71 & 33.63 & 32.00 \\
\hline $\begin{array}{l}\text { Square of } \\
\text { of Correlation }\end{array}$ & 0.544 & 0.408 & 0.154 & 0.866 \\
\hline Equation & $\begin{array}{c}\mathrm{Y}=0.145 \mathrm{X} \\
+25.38\end{array}$ & $\begin{array}{l}\mathrm{Y}=0.108 \mathrm{X} \\
+21.53\end{array}$ & $\begin{array}{l}\mathrm{Y}=0.062 \mathrm{X}+ \\
22.69\end{array}$ & $\begin{array}{l}\mathrm{Y}=0.142 \mathrm{X} \\
+19.83\end{array}$ \\
\hline $\begin{array}{l}\text { Prediscted C.S. } \\
\text { For 28 Day }\end{array}$ & 29.41 & 24.554 & 24.426 & 23.806 \\
\hline
\end{tabular}

FOR HOPPER NO:1 [M30 GRADE]

\begin{tabular}{|l|c|c|c|c|}
\hline Sr. No. & 9 & 10 & 11 & 12 \\
\hline 7 Day & 31.11 & 29.03 & 27.70 & 20.74 \\
\hline 28 Day & 41.78 & 32.15 & 34.97 & 28.75 \\
\hline 90Day of Coeff. & 43.26 & 35.70 & 40.59 & 40.00 \\
\hline $\begin{array}{l}\text { Square } \\
\text { of Correlation }\end{array}$ & $\begin{array}{l}\mathrm{Y}=0.595 \\
+33.777\end{array}$ & $\begin{array}{l}\mathrm{Y}=0.943 \\
+29.16\end{array}$ & 0.881 & 0.967 \\
\hline Equation & 37.074 & 31.26 & 32.56 & 26.56 \\
\hline $\begin{array}{l}\text { Prediscted C.S. } \\
\text { For 28 Day }\end{array}$ & $\begin{array}{l}\mathrm{Y}=0.140 \mathrm{X}+ \\
+20.64\end{array}$ \\
\hline
\end{tabular}

FOR HOPPER NO:2 [M20 GRADE]

\begin{tabular}{|l|c|c|c|c|}
\hline Sr. No. & $\mathbf{1 3}$ & $\mathbf{1 4}$ & $\mathbf{1 5}$ & $\mathbf{1 6}$ \\
\hline 7 Day & 20.15 & 18.50 & 18.37 & 17.77 \\
\hline 28 Day & 27.71 & 24.60 & 27.27 & 25.34 \\
\hline 90Day of Coeff. & 28.30 & 27.56 & 29.78 & 26.52 \\
\hline $\begin{array}{l}\text { Square } \\
\text { of Correlation }\end{array}$ & $\begin{array}{l}\mathrm{Y}=0.078 \mathrm{X} \\
+22.13\end{array}$ & $\begin{array}{l}\mathrm{Y}=0.095 \mathrm{X} \\
+19.58\end{array}$ & $\begin{array}{l}\mathrm{Y}=0.115 \mathrm{X}+ \\
20.33\end{array}$ & $\begin{array}{l}\mathrm{Y}=0.085+1 \\
9.63\end{array}$ \\
\hline Equation & 24.314 & 22.24 & 23.55 & 22.01 \\
\hline $\begin{array}{l}\text { Prediscted C.S. } \\
\text { For 28 Day }\end{array}$ & & & & 0.690 \\
\hline
\end{tabular}


FOR HOPPER NO:2 [M25 GRADE]

\begin{tabular}{|l|c|c|c|c|}
\hline Sr. No. & $\mathbf{1 7}$ & $\mathbf{1 8}$ & $\mathbf{1 9}$ & $\mathbf{2 0}$ \\
\hline 7 Day & 21.63 & 21.56 & 20.74 & 18.87 \\
\hline 28 Day & 35.85 & 32.60 & 32.23 & 29.04 \\
\hline 90Day & 36.89 & 35.71 & 37.04 & 31.41 \\
\hline $\begin{array}{l}\text { Square of Coeff. } \\
\text { of Correlation }\end{array}$ & 0.544 & 0.689 & 0.761 & 0.659 \\
\hline Equation & $\begin{array}{l}\mathrm{Y}=0.145 \mathrm{X} \\
+25.38\end{array}$ & $\begin{array}{l}\mathrm{Y}=0.143 \mathrm{X} \\
+23.99\end{array}$ & $\begin{array}{l}\mathrm{Y}=0.169 \mathrm{X}+ \\
22.94\end{array}$ & $\begin{array}{l}\mathrm{Y}=0.125 \mathrm{X} \\
+21.21\end{array}$ \\
\hline $\begin{array}{l}\text { Prediscted C.S. } \\
\text { For 28 Day }\end{array}$ & 29.44 & 27.994 & 27.672 & 24.71 \\
\hline
\end{tabular}

\section{FOR HOPPER NO:2 [M30 GRADE]}

\begin{tabular}{|l|c|c|c|c|}
\hline Sr. No. & 21 & 22 & 23 & 24 \\
\hline 7 Day & 31.11 & 29.50 & 28.50 & 21.70 \\
\hline 28 Day & 41.78 & 35.70 & 32.89 & 29.19 \\
\hline 90Day & 43.26 & 37.49 & 41.03 & 41.04 \\
\hline $\begin{array}{l}\text { Square of Coeff. } \\
\text { of Correlation }\end{array}$ & 0.595 & 0.693 & 0.989 & 0.978 \\
\hline Equation & $\begin{array}{l}\mathrm{Y}=0.118 \mathrm{X} \\
+33.77\end{array}$ & $\begin{array}{l}\mathrm{Y}=0.080 \mathrm{X} \\
+30.85\end{array}$ & $\begin{array}{l}\mathrm{Y}=0.146 \mathrm{X}+ \\
28.03\end{array}$ & $\begin{array}{l}\mathrm{Y}=0.223 \mathrm{X} \\
+21.33\end{array}$ \\
\hline $\begin{array}{l}\text { Prediscted C.S. } \\
\text { For 28 Day }\end{array}$ & 37.074 & 33.09 & 32.118 & 27.574 \\
\hline
\end{tabular}

HOPPER NO:3 [M20 GRADE]

\begin{tabular}{|l|c|c|c|c|}
\hline Sr. No. & $\mathbf{2 5}$ & $\mathbf{2 6}$ & $\mathbf{2 7}$ & $\mathbf{2 8}$ \\
\hline 7 Day & 20.15 & 19.00 & 19.11 & 18.07 \\
\hline 28 Day & 27.71 & 24.60 & 28.30 & 26.60 \\
\hline 90Day & 28.30 & 28.15 & 31.56 & 26.97 \\
\hline $\begin{array}{l}\text { Square of Coeff. } \\
\text { of Correlation }\end{array}$ & 0.548 & 0.843 & 0.730 & 0.520 \\
\hline Equation & $\begin{array}{l}\mathrm{Y}=0.078 \mathrm{X} \\
+22.13\end{array}$ & $\begin{array}{l}\mathrm{Y}=0.098 \mathrm{X} \\
+19.82\end{array}$ & $\mathrm{Y}=0.127 \mathrm{X}+$ & $\begin{array}{l}\mathrm{Y}=0.084 \mathrm{X} \\
+20.37\end{array}$ \\
\hline $\begin{array}{l}\text { Prediscted C.S. } \\
\text { For 28 Day }\end{array}$ & 24.314 & 22.564 & 24.546 & 22.722 \\
\hline
\end{tabular}

M25 GRADE

\begin{tabular}{|l|c|c|c|c|}
\hline Sr. No. & 29 & 30 & 31 & 32 \\
\hline 7 Day & 21.63 & 23.64 & 21.48 & 18.96 \\
\hline 28 Day & 35.85 & 36.60 & 33.56 & 31.26 \\
\hline 90Day of Coeff. & 36.89 & 39.26 & 38.07 & 32.45 \\
\hline $\begin{array}{l}\text { Square } \\
\text { of Correlation }\end{array}$ & $\begin{array}{l}\mathrm{Y}=0.145 \mathrm{X} \\
+25.38\end{array}$ & $\begin{array}{l}\mathrm{Y}=0.155 \mathrm{X} \\
+26.70\end{array}$ & $\begin{array}{l}\mathrm{Y}=0.171 \mathrm{X}+ \\
23.91\end{array}$ & $\begin{array}{l}\mathrm{Y}=0.129 \mathrm{X} \\
+22.14\end{array}$ \\
\hline Equation & 29.44 & 31.04 & 28.698 & 25.752 \\
\hline $\begin{array}{l}\text { Prediscted C.S. } \\
\text { For 28 Day }\end{array}$ & & & & 0.739 \\
\hline
\end{tabular}




\begin{tabular}{|l|c|c|c|c|}
\multicolumn{5}{|c|}{ M30 GRADE } \\
\hline Sr. No. & 33 & 34 & 35 & 36 \\
\hline 7 Day & 31.11 & 29.78 & 29.78 & 22.52 \\
\hline 28 Day & 41.78 & 33.64 & 36.89 & 32.45 \\
\hline 90Day & 43.26 & 43.26 & 42.97 & 41.18 \\
\hline $\begin{array}{l}\text { Square of Coeff. } \\
\text { of Correlation }\end{array}$ & 0.595 & 0.998 & 0.899 & 0.904 \\
\hline $\begin{array}{l}\text { Equation } \\
\text { Prediscted C.S. }\end{array}$ & 37.074 & 33.34 & 34.56 & 29.21 \\
For 28 Day & $\begin{array}{l}\mathrm{Y}=0.160 \mathrm{X} \\
+28.86\end{array}$ & $\begin{array}{l}\mathrm{Y}=0.145 \mathrm{X}+ \\
30.50\end{array}$ & $\begin{array}{l}\mathrm{Y}=0.205 \mathrm{X} \\
+23.47\end{array}$ \\
\hline
\end{tabular}

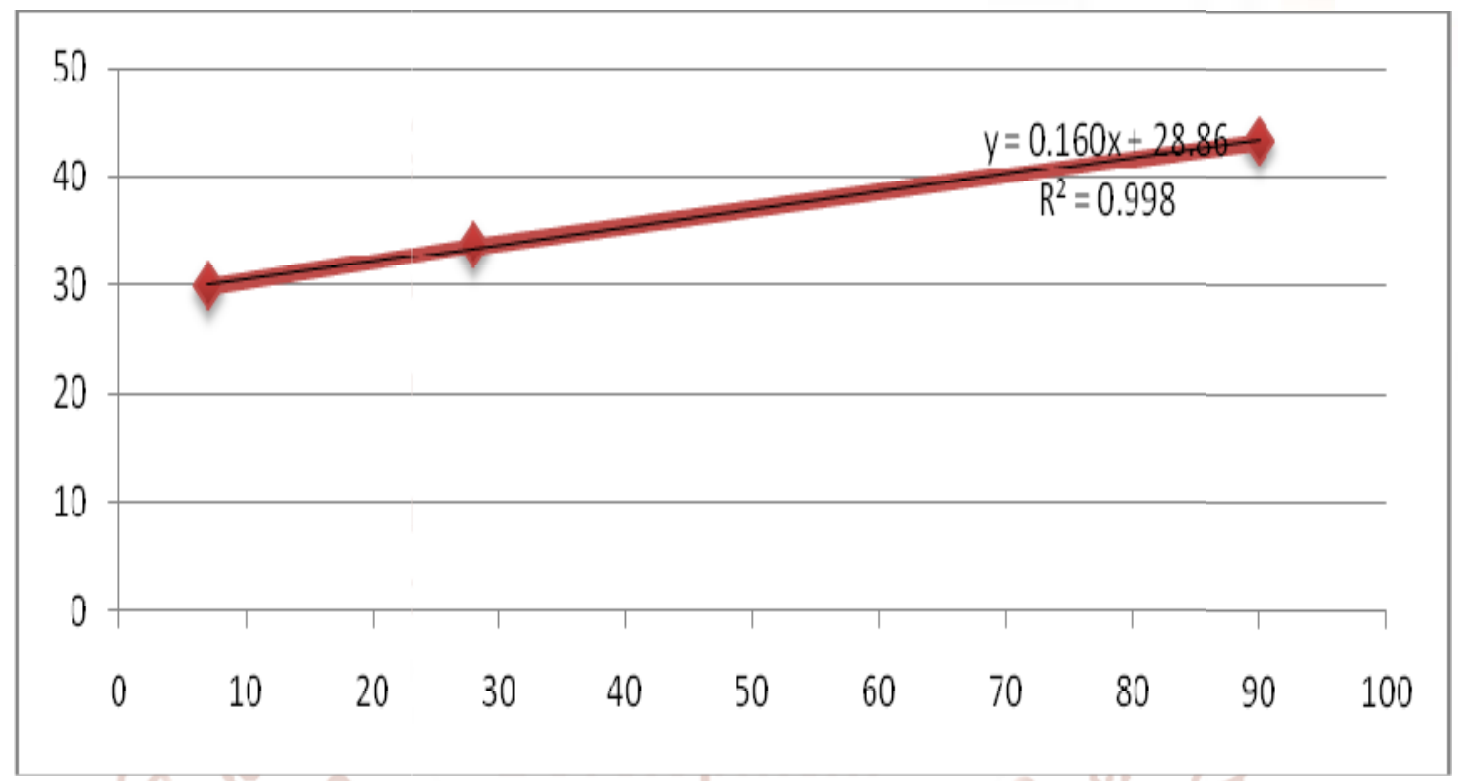

Fig.Days vs comp. Strength graph with equation and correlation coefficient.

\section{CONCLUSION}

\section{I}

1) A Correlation greater than 0.8 is generally described as strong, whereas a correlation less than 0.5 is generally described as weak.

1) Coefficient of correlation lies between -1 to 1 .

2) As we get coefficient of correlation by using regression analysis, more is the coefficient of correlation; more is the predicted compressive strength. If coefficient of correlation is nearer to 0.999 , predicted values became observed.

3) Also, for higher grade of concrete we have greater coefficient of correlation.

4) For M20 grade, minimum coefficient of correlation is 0.520 and maximum is 0.843

5) For M25 grade, minimum coefficient of correlation is 0.154 and maximum is 0.866

2) When it is nearer 0.999efficiency of getting accurate compressive strength of concrete is more.

3) Coefficient of correlation lies between 0.5 to 0.7 , results i.e. predicted values at 28 th day compressive strength have difference of $2 \mathrm{~N} / \mathrm{mm} 2$ to $3 \mathrm{~N} / \mathrm{mm} 2$ than observed values.

6) For M30 grade, minimum coefficient of correlation is 0.544 and maximum is 0.989 .

\section{III}

1) By deriving equation, we can predict the values of compressive strength at any curing days like 28th day.

2) We get more deviated equation when coefficient of correlation is less. 
3) And relatively accurate equation when we have large coefficient of correlation.

4) Eg. In 34th result. Observed compressive strength is 33.64 and that of predicted value is 33.34 and having coefficient of correlation 0.998 when Fly ash mixing is $12.5 \%$.

\section{SUMMARY}

In this way, regression analysis helps to predict compressive strength at any stage of curing days and correlation between compressive strength at different stages.

\section{REFERENCES}

1) Suhad M.A., Mathematical model for the prediction of cement compressive strength at the ages of $7 \& 28$ days within 24 hours , MSc Thesis, Al-Mustansiriya University, college of engineering, civil engineering department, 2001.

2) Sandorpopovics, Analysis of Concrete Strength Versus Water-Cement Ratio Relationship , ACI Material Journal,Vol.87, No.5, nt SeptemberOctober 1990, Pp.517-529

3) Kheder G.F.,AL-Gabban A.M. \&Suhad M.A., Mathematical model for the prediction of cement compressive strength at the ages of $7 \& 28$ days within 24 hour materials and structures2003. 36: 693-701
4) Hasan M.M. and Kabir A., Prediction of Compressive Strength of Concrete from Early Age Test Result . Proceedings of 4thAnnual Paper Meet and 1st Civil Engineering Congress, Dhaka,Bangladesh, December 22-24, 2011, pp. 17

5) Ahsanul Kabir, Monjurul Hasan2 AND MD. Khasro Miah, Predicting 28 Days Compressive Strength of Concrete from 7 Days Test Result ,Bangladesh University of Engg. \& Tech. /Dept. of Civil Engineering, Dhaka, Bangladesh

6) M. F. M. Zain1, Suhad M. Abd, K. Sopian, M. Jamil, Che-Ani A.I , Mathematical Regression Model for the Prediction of Concrete Strength ,Faculty of Engineering and Built Environment, Solar Energy Research Institute, UniversitiKebangsaan Malaysia, 43600 UKM Bangi, Selangor DarulEhsan, Malaysia .

7) Palika Chopra, DR. $R \quad K$ Sharma, DR. Maneekkumar , Regression models for the prediction of compressive strength of concrete with and without fly ash, thapar university , Patiala-147004.

8) Dinesh.W: Gawatre \& V.G.Meshram, Study Of Various Fineness Of Fly Ash As A Partial Replacement Of Cement In Concrete International Journal of Scientific \& Engineering Research Volume 4, Issue 1, January-2013 .PP 01-07 